\title{
Algebraic Properties of Program Integration
}

\author{
Thomas Reps \\ Computer Sciences Department \\ University of Wisconsin-Madison \\ 1210 W. Dayton Street \\ Madison, WI 53706 USA
}

The need to integrate several versions of a program into a common one arises frequently, but it is a tedious and time consuming task to merge programs by hand. The program-integration algorithm recently proposed by Horwitz, Prins, and Reps provides a way to create a semantics-based tool for integrating a base program with two or more variants. The integration algorithm is based on the assumption that any change in the behavior, rather than the text, of a program variant is significant and must be preserved in the merged program. An integration system based on this algorithm will determine whether the variants incorporate interfering changes, and, if they do not, create an integrated program that includes all changes as well as all features of the base program that are preserved in all variants. To determine this information, the algorithm employs a program representation that is similar to the program dependence graphs that have been used previously in vectorizing and parallelizing compilers.

This paper studies the algebraic properties of the program-integration operation. To do so, we first modify the integration algorithm by recasting it as an operation on a Brouwerian algebra constructed from sets of dependence graphs. (A Brouwerian algebra is a distributive lattice with an operation $a-b$ characterized by $a-b \subseteq c$ iff $a \subseteq b \cup c$.) In this algebra, the program-integration operation can be defined solely in terms of $u$, $n$, and - . By making use of the rich set of algebraic laws that hold in Brouwerian algebras, the paper establishes a number of the integration operation's algebraic properties.

\section{INTRODUCTION}

The algorithm recently proposed by Horwitz, Prins, and Reps [5], which we will refer to as the $H P R$ algorithm, provides the basis for a tool to integrate (combine) program variants automatically. Given a program $B$ ase and two variants $A$ and $B$, the HPR algorithm makes use of knowledge of the programming language to determine whether the changes made to $B$ ase to produce $A$ and $B$ interfere; if there is no interference, the algorithm produces a merged program $M$. One of the virtues of the HPR algorithm is that it is possible to characterize the execution behavior of program $M$ in terms of the behaviors of $B a s e, A$, and $B$. (By the execution behavior of a program for some initial state, we mean the sequence of values produced at each program component when the program is run on that state.) Changes in the behavior of $A$ and $B$ with respect to Base-rather than changes in their text-are detected and preserved in the integrated program, along with the unchanged behavior of all three [11]. Although it is undecidable to determine whether a program modification actually leads to a change in execution behavior, it is possible to determine a safe approximation by comparing $A$ and $B$ with Base, using a program representation that is similar to the program dependence graphs used in vectorizing and parallelizing compilers $[1,6]$.

One aspect of the HPR algorithm that has not been previously addressed is its algebraic properties. These are of interest when dealing with compositions of integrations. For example, if three variants of a given base program are to be integrated by a pair of (two-variant) integrations, it is important to know

This work was supported in part by a David and Lucile Packard Fellowship for Science and Engineering, by the National Science Foundation under grant DCR-8552602, by the Defense Advanced Research Projects Agency, monitored by the Office of Naval Research under contract N00014-88-K-0590, as well as by grants from IBM, $\mathrm{DEC}$, and Xerox. 
whether there is a law of associativity to guarantee that it does not matter which two variants are integrated first. (Such a law does hold, and is discussed in Section 4.)

Although the program-integration problem addressed by the HPR algorithm is a greatly simplified one and the algorithm's capabilities are severely limited-the algorithm is developed for a simple language in which expressions contain scalar variables and constants, and the only statements are assignment statements, conditional statements, and while-loops-recent research has made progress towards extending the set of language constructs to which the algorithm is applicable $[3,4]$, as well as incorporating some alternative techniques $[15,16]$. Our hope is that such extensions and modifications will share with the basic HPR algorithm a common set of algebraic properties. However, we would like to avoid having to re-prove that each property holds every time we enhance our techniques. Instead, we would like to have a framework that would not only let us establish the algebraic properties of program integration, but would also allow us to show that a new algorithm possesses these properties merely by demonstrating that the algorithm meets the conditions of the framework. This paper uses lattice theory to provide such a framework.

A novel feature of our study is the use of Brouwerian algebra, rather than, for example, Boolean algebra or relational algebra. A Brouwerian algebra [7] is a distributive lattice with a pseudodifference operation, $a-b$, characterized by $a-b \subseteq c$ iff $a \subseteq b \cup c$ (see Section 3). The connection between program integration and Brouwerian algebra is made as follows: we introduce a Brouwerian algebra constructed from sets of dependence graphs; in this algebra, the program-integration operation can be expressed solely in terms of the operations $u, n$, and $\doteq$.

The contributions of the paper can be summarized as follows:

(1) The paper establishes a number of algebraic properties that hold for the integration operation. These investigations make use of the rich set of algebraic laws that hold in Brouwerian algebras.

(2) The paper provides a lattice-theoretic framework for studying the common properties of different integration algorithms. The operation we define to integrate elements of a Brouwerian algebra is

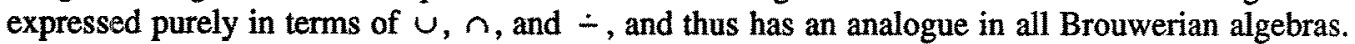
In Sections 4,5 , and 6 , the properties of the integration operation are established using only algebraic identities and inequalities, and thus the results obtained hold for all Brouwerian algebras. Consequently, to show that a proposed program-integration algorithm shares these properties, one merely has to show that the algorithm can be cast as an integration operation in some Brouwerian algebra.

(3) The paper describes how to eliminate a restriction that is part of the HPR algorithm. The HPR algorithm assumes that a special program editor is used to create the program variants from the base program: the editor provides a tagging capability so that common statements and predicates can be identified in different versions. As discussed in Section 7 , it is possible to do away with this restriction and use Brouwerian algebras whose elements are dependence graphs that do not have tags on their components. Thus, in principle it is no longer necessary for program integration to be supported by a closed system; programs can be integrated even if they are created using ordinary text editors.

(4) The paper identifies a new criterion for program integration, based on the operation in our framework that is the dual of the integration operation.

The paper is organized into eight sections. Section 2 provides a brief introduction to the HPR algorithm. Section 3 defines Brouwerian algebras, and introduces a particular Brouwerian algebra constructed from sets of dependence graphs. Section 4 defines the operation to integrate elements of a Brouwerian algebra. For the dependence-graph algebra discussed in Section 3, this operation corresponds very closely to the HPR algorithm. Section 5 concerns the operation that is the dual of the integration operation, and shows how the two operations are related. Section 6 discusses an application of program integration. Section 7 describes the relationship of the work described in the paper to pre- 
vious work; in particular, it compares the integration operation in the dependence-graph algebra with the HPR algorithm. Section 8 briefly describes an implementation of a program-integration tool that incorporates the ideas presented in the paper.

Except in Section 5, all proofs are omitted. Section 5 illustrates the power of the algebraic approach for establishing integration properties; proofs of all other properties cited can be found in [13].

\section{OVERVIEW OF THE HPR ALGORITHM FOR PROGRAM INTEGRATION}

The HPR algorithm applies to a simplified programming language with the following characteristics: expressions contain only scalar variables and constants; statements are either assignment statements, conditional statements, while loops, or a restricted kind of "output statement" called an end statement, which can only appear at the end of a program. An end statement names zero or more of the variables used in the program. The variables named in the end statement are those whose final values are of interest to the programmer; when execution terminates, the final state is defined on only those variables in the end statement. Thus a program is of the form

program

list-of-statements
end $\left(i d^{*}\right)$.

Variables that are used before being defined get their value from the initial execution state.

A full description of the HPR algorithm can be found in [5]; because of space limitations, the description given here will be confined to the case of straight-line code (with end statements). We wish to stress that this does not reflect a limitation of the approach described in the paper; our approach applies in toto to the more general language handled by the full HPR algorithm.

The integration of $A$ and $B$ with respect to $B a s e$, which will be denoted by $A$ [Base $] B$, requires combining three structures, $\Delta(A, B a s e), \Delta(B, B a s e)$, and Pre $(A, B a s e, B)$, where $\Delta(A, B a s e)$ and $\Delta(B, B$ ase $)$ represent potentially changed computations of $A$ and $B$ with respect to $B a$ se, respectively, and Pre $(A, B$ ase,$B)$ represents computations that are preserved in all three. To determine this information, the algorithm employs graphs that represent the dependences between program elements. For straight-line code, the vertices represent the program's assignment statements and final uses of variables in the program's end statement; the edges represent write/read dependences (also known as flow dependences or def-use chains): an edge runs from vertex $v$ to vertex $w$ if (1) $v$ assigns to a variable $x$, (2) $w$ uses variable $x,(3) v$ precedes $w$, and (4) there is no intervening assignment to $x$.

To find potentially changed and preserved computations, the algorithm makes use of an operation called slicing [8,14]. A slice of a dependence graph with respect to vertex $v$ consists of all elements on paths to $v$, which is exactly the set of elements that can affect the value produced at $v$ (i.e., that can affect the behavior of $v$ ). For example, when the graph for the program shown below on the left is sliced with respect to the final use of $y$ in the program's end statement, the result is a graph that corresponds to the program shown on the right.

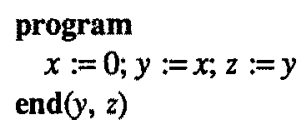

The slice with respect to a set of vertices is the union of the slices taken with respect to the set's members. The slice with respect to a graph $H$ is defined to be the slice taken with respect to the vertex set of $H$.

The significance of a slice is that it captures a portion of a program's behavior in the sense that, for any initial state on which the program halts, the program and the slice compute the same sequence of values for each element of the slice [11]. 
THEOREM. (SLICING THEOREM [11]). Let $Q$ be a slice of program $P$ with respect to a set of vertices. If $\sigma$ is a state on which $P$ halts, then (1) $Q$ halts on $\sigma$, (2) $P$ and $Q$ compute the same sequence of values at each program point of $Q$, and (3) The final states agree on all variables defined in the final state of $Q$.

The HPR algorithm assumes that a special program editor-providing a tagging capability so that corresponding statements can be identified in different versions-is used to create the program variants from the base program. Such correspondences are necessary to determine $\Delta(A, B$ ase $), \Delta(B, B$ ase $)$, and $\operatorname{Pre}(A, B a s e, B): \triangle(A, B a s e)$ consists of the slices of $A$ with respect to all vertices $v$ such that either vertex $v$ is not in Base, or the slice of Base with respect to $v$ differs from the slice of $A$ with respect to $v ; \Delta(B, B a s e)$ is defined similarly for $B ; \operatorname{Pre}(A, B a s e, B)$ consists of the slices that are identical in all three graphs. The merged program created by the HPR algorithm corresponds to the graph obtained by taking the union of all these slices.

Example. The following example illustrates the HPR algorithm. The tags on statements are noted between square brackets.

\begin{tabular}{||ccccccc|}
\hline$A$ & Base & $B$ & $\Delta(A$, Base $)$ & Pre $(A$, Base,$B)$ & $\Delta(B$, Base $)$ & $A[$ Base $] B$ \\
\hline program & program & program & $\varnothing$ & program & program & program \\
{$[1] x:=0$} & {$[1] x:=0 ;$} & {$[1] x:=0 ;$} & & {$[1] x:=0$} & {$[1] x:=0 ;$} & {$[1] x:=0 ;$} \\
end $(x)$ & {$[2] y:=x$} & {$[2] y:=x ;$} & & end $(x)$ & {$[2] y:=x ;$} & {$[2] y:=x ;$} \\
& end $(x, y)$ & $\begin{array}{l}{[3] z:=y} \\
\text { end }(x, y, z)\end{array}$ & & & {$[3] z:=y$} & {$[3] z:=y$} \\
& & & & end $(z)$ & end $(x, z)$ \\
\hline
\end{tabular}

This example illustrates one subtlety of the HPR algorithm: an insertion made in one integrand can "override" a deletion in the other integrand. In the example given above, the insertion of $z:=y$ in integrand $B$ overrides the deletion of $y:=x$ from integrand $A$ because $z:=y$ uses the value assigned to $y$ by $y:=x$. Note that the deletion from $A$ of the final use of $y$ does not get overridden.

\section{USING LATTICE THEORY TO DESCRIBE PROGRAM INTEGRATION}

To understand the considerations that led us to recast the HPR algorithm as an operation in a Brouwerian algebra, consider how Pre $(A, B a s e, B)$ was characterized above: "Pre $(A, B a s e, B)$ consists of the slices that are identical in all three graphs." This suggests that Pre $(A, B a s e, B)$ is the meet of $A, B a s e$, and $B$ in a lattice ${ }^{1}$ of dependence graphs ordered by "is-a-slice-of," the meet operation being "greatest common slice." (Here the term "dependence graph" refers to the more general kind used in the full HPR algorithm, not just the kind for straight-line code described in Section 2.) The fact that $\Delta(A, B a s e)$ and $\Delta(B, B a s e)$ are then combined with Pre $(A, B a s e, B)$ suggests that this combination is a join operation. The terms $\Delta(A, B a s e)$ and $\Delta(B, B a s e)$ themselves suggest that some sort of difference operation exists for elements of the lattice.

Complicating matters is the fact that the set of dependence graphs ordered by is-a-slice-of is a lower semi-lattice, but not a lattice (i.e., it has a meet operation but no join operation). In particular, the

${ }^{1}$ A lattice is an algebra $(L, \cup, n)$, where $L$ is a set of elements that is closed under $\cup$ (join) and $\cap$ (meet), and for all $a, b$, and $c$ in $L$ the following axioms are satisfied:
$a \cup a=a$
$a \cup b=b \cup a$
$a \cap a=a$
$a \cap b=b \cap a$
$(a \cup b) \cup c=a \cup(b \cup c)$
$(a \cap b) \cap c=a \cap(b \cap c)$
$a \cup(a \cap b)=a$
$a \cap(a \cup b)=a$

The symbol $\subseteq$ is used to denote the partial order on the elements of $L$ given by $a \subseteq b$ iff $a \cap b=a$. All lattices considered in this paper have a least and a greatest element, which are denoted by $\perp$ and $T$, respectively. 
operation of unioning two dependence graphs, which is used in the HPR algorithm to combine the dependence graphs that represent $\Delta(A, B a s e), \Delta(B, B a s e)$, and $\operatorname{Pre}(A, B a s e, B)$, is not a join operation. To see this, consider the union of the dependence graphs for the programs $a$ and $b$ shown below.

\section{$a$}

\section{program}

[1] $x:=0 ;[2] y:=x$ end $(y)$

\section{$b$}

program

$[2] y:=x ;[3] z:=y$

end $(z)$

The result of $a \cup b$ is a dependence graph that corresponds to the program

program

$[1] x:=0 ;[2] y:=x ;[3] z:=y$

end $(y, z)$.

For $\cup$ (graph union) to be a join operation, both $a$ and $b$ have to be slices of $a \cup b$. However, although both $a$ and $b$ are subgraphs of $a \cup b$, only $a$ is a slice of $a \cup b$. The graph for $b$ is not a slice of $a \cup b$ because in $a \cup b$ vertex $y:=x$ is the target of a flow edge whose source is $x:=0$, which "corrupts" the slices of $a \cup b$ with respect to $y:=x, z:=y$, and the final use of $z$.

The solution involves defining a lattice whose elements consist of sets of slices, and performing operations on these sets; in particular, the join operation is set union. However, in order for set intersection to capture common slices, it is necessary to work with sets having a particular structure (rather than arbitrary sets of slices). Similarly, ordinary set difference turns out not to capture the notion of $\Delta(A, B$ ase $)$ and $\Delta(B, B$ ase $)$ from the HPR algorithm, but a variation on set difference that takes into account the ordering relation on slices can be used instead.

Definition. Let $G$ be the set of well-formed program dependence graphs, and let the relation symbol $\leq$ denote the relation "is-a-slice-of," (i.e., $x \leq y$ means that graph $x$ is a slice of graph $y$ ).

(i) Define set $G_{1}$, the set of all program dependence graphs that are single-point slices, to be $G_{1} \triangleq\{g \in G \mid \exists x \in V(g)$ such that $(g / x)=g\}$, where $g / x$ denotes the slice of $g$ with respect to vertex $x$.

(ii) Define set $D C S$, the set of all downwards-closed sets of single-point slices, to be $D C S \triangleq\left\{S \in P\left(G_{1}\right) \mid \forall x \in G_{1}\right.$ if $\exists s \in S$ such that $x \leq s$ then $\left.x \in S\right\}$, where $P\left(G_{1}\right)$ denotes the power set of $G_{1}$.

(iii) For all $x, y \in D C S$, define the operation pseudo-difference, denoted by $x-y$, to be $x-y \triangleq\left\{z \in G_{1} \mid \exists p \in(x-y)\right.$ such that $\left.z \leq p\right\}$, where $x-y$ denotes the set difference between $x$ and $y$. That is, $x-y$ is the downwards closure of $x-y$.

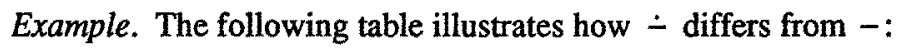

\begin{tabular}{|c|c|c|c|c|c|}
\hline$a$ & $b$ & $a-b$ & $a \div b$ & $b-a$ & $b \div a$ \\
\hline (program) & (program, program & $\varnothing(=1)$ & $\varnothing(=\perp)$ & [program & [program, program \\
\hline$\left\{\begin{array}{c}x:=0 \\
\text { end }(0\end{array}\right\}$ & $\left\{\begin{array}{cc}x:=0 & x:=0 \\
\text { end }(0) & y:=x \\
& \text { end } 0\end{array}\right.$ & & & $\left\{\begin{aligned} & x:=0 \\
& y:=x \\
& \text { end } 0\end{aligned}\right.$ & $\left\{\begin{array}{cc}x:=0 & x:=0 \\
\text { end } 0 & y:=x \\
& \text { end } 0\end{array}\right.$ \\
\hline
\end{tabular}

This structure-the set DCS together with the operations of set union, set intersection, and pseudodifference-is an instance of what is known as a Brouwerian algebra (defined below). The benefit of this fact is that Brouwerian algebras have a rich set of algebraic laws, consisting of identities and inequalities (see the Appendix). These laws provide a convenient way to establish the integration operation's algebraic properties through simple formula manipulations. (For examples, see Section 5.) 
Definition. A Brouwerian algebra [7] is an algebra $(L, \cup, \cap,-, T)$ where

(i) $(L, \cup, \cap)$ is a lattice with greatest element $T$.

(ii) $L$ is closed under - .

(iii) For all $a, b$, and $c$ in $L, a-b \subseteq c$ iff $a \subseteq b \cup c$.

It can be shown that $L$ has a least element, given by $\perp=T-T$, and that $(L, \cup, \cap)$ is distributive. ${ }^{2}$ THEOREM. (DCS, $\left.\cup, \cap,-, G_{1}\right)$ is a Brouwerian algebra, where $\cup$ is set union and $\cap$ is set intersection.

\section{INTEGRATION OF ELEMENTS OF A BROUWERIAN ALGEBRA}

We now introduce a ternary operation on elements of a Brouwerian algebra that, for the dependencegraph algebra discussed in Section 3, corresponds very closely to the HPR algorithm. (The relationship between integration in the dependence-graph algebra and the HPR algorithm is addressed further in Section 7.)

Definition. The integration of elements $a$ and $c$ with respect to element $b$ is the element $a[b] c$ defined by $a[b] c \triangleq(a-b) \cup(a \cap b \cap c) \cup(c-b)$. If $a[b] c=m$, we refer to element $b$ as the base, elements $a$ and $c$ as the integrands, and element $m$ as the result of the integration.

Example. The table shown in Figure 1, which indicates what slices are members of the sets $A, B$ ase, $B, A-B$ ase, $A \cap B a s e \cap B, B-B a s e$, and $A[B a s e] B$, illustrates the integration operation (in the dependence-graph algebra) for the example from Section 2 . This produces the same result that was obtained in Section 2 with the HPR algorithm; as shown in Figure 1, the set of slices computed for $A[$ Base $] B$ corresponds to the program

program

$x:=0 ; y:=x ; z:=y$

$\operatorname{end}(x, z)$.

Because the integration operation is defined solely in terms of $u, n$, and - , it has an analogue in all Brouwerian algebras, not just the dependence-graph algebra from Section 3. Because we will study its properties strictly from an algebraic standpoint, our results apply to this operation in all Brouwerian algebras. It is not difficult to show that the following basic properties hold for the integration operation:

$a[a] b=b$

$a[\perp] b=a \cup b$

$$
\begin{aligned}
& a[b] a=a \\
& a[T] b=a \cap b
\end{aligned}
$$

${ }^{2}$ A Brouwerian algebra is quite similar, but not identical, to a Boolean algebra. The relationship between
Boolean and Brouwerian algebras can be characterized as follows [7]: for all elements $a$, define the Brouwerian
complement by $\neg a \triangleq T-a$; a Brouwerian algebra is Boolean iff $\neg \neg a=a$. Because Boolean algebras are
Brouwerian algebras, but not vice versa, some of the properties that hold in Boolean algebras do not hold in
Brouwerian algebras. For example, the laws for distributing $\doteq$ over $\cup$ and $\cap$ in Brouwerian algebra are
somewhat different from the laws for distributing - over $\cup$ and $n$ in Boolean algebra. Two of the laws are the
same: $(16)(b \dot{-a}) \cup(c-a)=(b \cup c) \div a$ (17) $(c \dot{-a}) \cup(c \dot{-} b)=c \dot{\sim}(a \cap b)$

However, the laws for distributing - through $n$ on the left and $\cup$ on the right are weaker:

(29) $(a \cap b) \div c \subseteq(a-c) \cap(b-c) \quad(32) c \div(a \cup b) \subseteq(c-a) \cap(c-b)$

One can show by means of examples that the inequalities in laws (29) and (32) are, at times, strict. 


\begin{tabular}{|c|c|c|c|c|c|c|}
\hline \multirow[b]{2}{*}{ Term } & \multicolumn{6}{|c|}{ Slice } \\
\hline & $\begin{array}{c}\text { program } \\
x:=0 \\
\text { end }()\end{array}$ & $\begin{array}{c}\text { program } \\
x:=0 \\
\text { end }(x)\end{array}$ & $\begin{array}{c}\text { program } \\
x:=0 \\
y:=x \\
\text { end }()\end{array}$ & $\begin{array}{c}\text { program } \\
x:=0 \\
y:=x \\
\text { end }(y)\end{array}$ & $\begin{array}{c}\text { program } \\
x:=0 \\
y:=x \\
z:=y \\
\text { endo }\end{array}$ & $\begin{array}{r}\text { program } \\
x:=0 \\
y:=x \\
z:=y \\
\text { end }(z)\end{array}$ \\
\hline$A$ & $\mathrm{x}$ & $\mathrm{x}$ & & & & \\
\hline Base & $\mathrm{x}$ & $\mathrm{x}$ & $x$ & $\mathrm{x}$ & & \\
\hline $\begin{array}{c}B \\
A-B \text { Base }\end{array}$ & $\mathbf{x}$ & $x$ & $\mathrm{x}$ & $\mathrm{x}$ & $x$ & $x$ \\
\hline$A \cap$ Base $\cap B$ & $\mathrm{x}$ & $\mathrm{x}$ & & & & \\
\hline$B-$ Base & $\mathbf{x}$ & & $\mathrm{x}$ & & $\mathbf{x}$ & $\mathrm{x}$ \\
\hline$A[$ Base $] B$ & $\mathrm{x}$ & $\mathrm{x}$ & $\mathbf{x}$ & & $\mathrm{x}$ & $\mathrm{x}$ \\
\hline
\end{tabular}

Figure 1. Member slices of the sets $A, B$ ase, $B, A-B$ ase, $A \cap B$ ase $\cap B, B-B$ ase, and $A$ [Base $] B$.
$a[b]\left(x_{1} \cup x_{2}\right)=a[b] x_{1} \cup a[b] x_{2}$
$a[b] c$ is monotonic in $a$
$a[b] c-b=(a-b) \cup(c-b)$
$a[b]\left(x_{1} \cap x_{2}\right) \subseteq a[b] x_{1} \cap a[b] x_{2}$
$a[b] c$ is antimonotonic in $b$
$a[b] c-c=(a-b) \div c$

The integration operation can be generalized to integrate multiple variants with a given base element simultaneously.

Definition. The simultaneous integration of elements $x_{1}, x_{2}, \cdots, x_{n}$ with respect to element $b$ is the element $\left(x_{1}[b] x_{2}, \cdots, x_{n}\right)$ defined by

$\left(x_{1}[b] x_{2}, \cdots, x_{n}\right) \triangleq\left(x_{1}-b\right) \cup\left(x_{2}-b\right) \cup \cdots \cup\left(x_{n} \dot{-1}\right) \cup\left(x_{1} \cap x_{2} \cap \cdots \cap x_{n} \cap b\right)$.

The following associativity theorem shows that a three-variant simultaneous integration can be done as a succession of two-variant integrations (in several different ways).

THEOREM (ASSOCIATTVITY THEOREM).

$(x[b] y)[b] z=x[b](y[b] z)=(x[b] z)[b] y=(x[b] y)[b](x[b] z)=(x[b] y)[x](x[b] z)=(x[b] y, z)$.

\section{AN ALTERNATIVE WAY TO PERFORM INTEGRATION}

This section concerns a new criterion for program integration, based on the operation that is the dual of the integration operation. After introducing a few new concepts that are needed to define the dual operation, we show how the two operations are related.

Definition. For all $x, y \in D C S$, define the operation quotient, denoted by $x \div y$, to be $x \div y \triangleq\left\{z \in G_{1} \mid \forall p \in(y-x) p \leq z\right\}$. That is, $x \div y$ is the complement of the upwards closure of $y-x$.

In the definition of $x \div y$, elements of the set $y-x$ represent forbidden (sub-)slices of members of $x \div y$. 
Example. The following table illustrates,$-\llcorner$, and $\div$ :

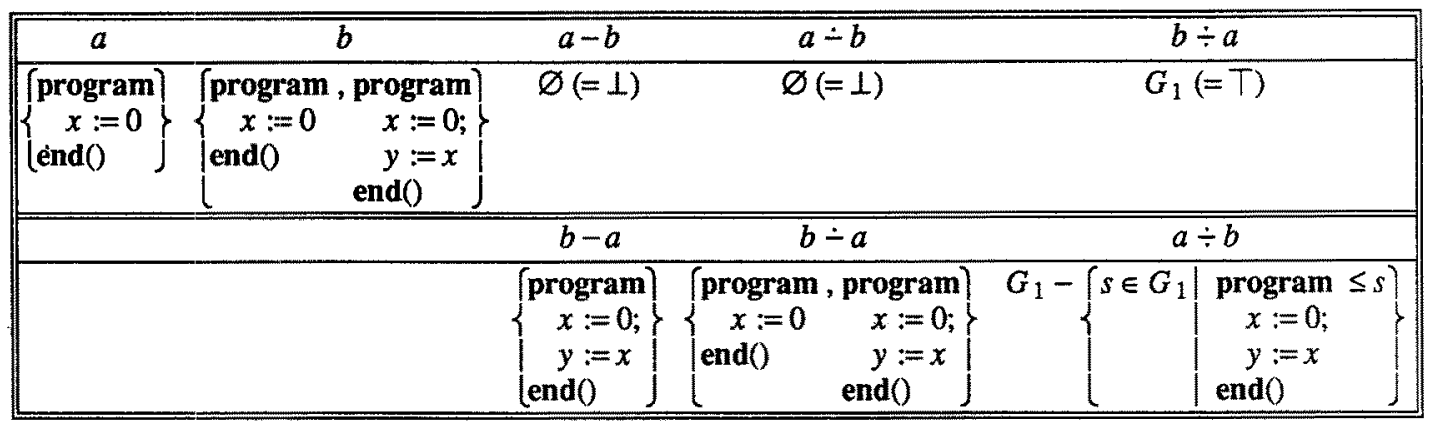

Note that because $b-a$ is the singleton set

$\left\{\begin{array}{l}\text { program } \\ x:=0 ; y:=x \\ \text { end }()\end{array}\right\}$

$a \div b$ is the (infinite) set of all single-point slices that do not contain the (sub-)slice

program

$x:=0 ; y:=x$

end().

Definition. A double Brouwerian algebra [7] is an algebra $(L, \cup, \cap,-, \div, \top)$ where both $(L, \cup, \cap,-, T)$ and $(L, \cap, \cup, \div, \top \div T)$ are Brouwerian algebras. In particular,

(i) $L$ is closed under $\div$.

(ii) For all $a, b$, and $c$ in $L, a \div b \supseteq c$ iff $a \supseteq b \cap c$.

THEOREM. (DCS, $\left.\cup, \cap,-, \div, G_{1}\right)$ is a double Brouwerian algebra, where $\cup$ is set union and $\cap$ is set intersection.

Because the dependence-graph algebra is a double Brouwerian algebra, it is possible to perform integration using the dual of the operation $a[b] c$.

Definition. The dual integration of elements $a$ and $c$ with respect to element $b$ is the element $a\{b\} c$ defined by $a\{b\} c \triangleq(a \div b) \cap(a \cup b \cup c) \cap(c \div b)$.

Note that, in the dependence-graph algebra, if $a, b$, and $c$ are all finite sets, then $a \cup b \cup c$ is finite. Hence even though $a \div b$ and $c \div b$ may be infinite, $a\{b\} c$ is guaranteed to be finite.

We now investigate how $a[b] c$ and $a\{b\} c$ are related; the theorem proven below shows that $a[b\} c$ is always less than or equal to $a[b] c$. We then give an example for which strict inequality holds.

LEMMA. $(a \dot{-} b) \cup(a \cap b \cap c)=(a \dot{-b}) \cup(a \cap c)$.

PROOF.

$$
\begin{aligned}
(a-b) \cup(a \cap b \cap c) & =((a-b) \cup(a \cap b)) \cap((a-b) \cup c) \\
& =a \cap((a-b) \cup c) \\
& =(a \cap(a-b)) \cup(a \cap c) \\
& =(a-b) \cup(a \cap c)
\end{aligned}
$$


COROLLARY, $a[b] c=(a-b) \cup(a \cap c) \cup(c-b)$.

PROOF. Immediate from the definition of $a[b] c$ and the preceding proposition.

THEOREM. $a[b] c \subseteq a[b] c$.

PROOF.

$$
\begin{aligned}
a[b] c & =(a-b) \cup(a \cap c) \cup(c-b) & & \text { by the preceding corollary } \\
& =((a \cup c)-b) \cup(a \cap c) & & \text { by (16) } \\
& =(a \cup((a \cup c) \div b)) \cap(c \cup((a \cup c)-b)) & & \\
& =(a \cup(a-b) \cup(c-b)) \cap(c \cup(a-b) \cup(c-b)) & & \text { by (16) } \\
& =(a \cup(c-b)) \cap(c \cup(a-b)) & & \text { by (13) }
\end{aligned}
$$

By the dual derivation, we have $a\{b\} c=(a \cap(c \div b)) \cup(c \cap(a \div b))$.

$$
\begin{aligned}
& a\{b\} c-a[b] c=[(a \cap(c \div b)) \cup(c \cap(a \div b))] \div[(a \cup(c \dot{-}))) \cap(c \cup(a-b))] \\
& =((a \cap(c \div b)) \div[(a \cup(c-b)) \cap(c \cup(a \div b))]) \\
& \cup((c \cap(a \div b)) \div[(a \cup(c \div b)) \cap(c \cup(a \div b))]) \quad \text { by (16) } \\
& =((a \cap(c \div b)) \div(a \cup(c \div b))) \cup((a \cap(c \div b)) \div(c \cup(a \div b))) \text { by }(17) \\
& \cup((c \cap(a \div b)) \div(a \cup(c-b))) \cup((c \cap(a \div b)) \div(c \cup(a \div b))) \\
& =((a \cap(c \div b))-(c \cup(a \div b))) \cup((c \cap(a \div b)) \div(a \cup(c-b))) \text { by }(4) \\
& (a \cap(c \div b)) \div(c \cup(a \div b))=((a \cap(c \div b)) \div(a \div b)) \div c \\
& \subseteq((c \div b) \cap(a-(a-b))) \div c \\
& \subseteq((c \div b) \cap(a \cap b))-c \\
& =(((c \div b) \cap b) \cap a) \div c \\
& =(c \cap b \cap a) \div c \\
& =1
\end{aligned}
$$

Similarly, $\quad(c \cap(a \div b)) \div(a \cup(c-b))=\perp$. Hence, $a\{b] c-a[b] c=\perp$, and thus, by (4), $a\{b\} c \subseteq a[b] c$.

Example. Returning once again to the integration example from Sections 2 and 4, Figure 2 shows that the inequality in the above theorem is, at times, strict; the programs shown in Figure 2 have the property that $A\{$ Base $\} B \subset A[B$ ase $] B$. In this example, only the maximal single-point slices in each set are listed, rather than the entire downwards-closed set. For instance, the program listed as $B$,

\section{program}

$x:=0 ; y:=x ; z:=y$

end $(x, y, z)$,

stands for the set

$\left\{\begin{array}{cccccc}\text { program } & \text { program } & \text { program } & \text { program } & \text { program } & \text { program } \\ x:=0 & x:=0 & x:=0 ; & x:=0 ; & x:=0 ; & x:=0 ; \\ \text { end }() & \text { end }(x) & y:=x & y:=x & y:=x ; & y:=x ; \\ & & \text { end }() & \text { end }(y) & z:=y & z:=y \\ & & & & \text { end }() & \text { end }(z)\end{array}\right\}$

\footnotetext{
${ }^{3}$ The laws used to justify proof steps are listed in the Appendix.
} 


\begin{tabular}{|c|c|c|c|c|}
\hline \multicolumn{2}{|c|}{$A$} & Base & $B$ & \\
\hline \multicolumn{2}{|c|}{$\begin{array}{c}\text { program } \\
x:=0 \\
\text { end }(x)\end{array}$} & $\begin{array}{c}\text { program } \\
x:=0 \\
y:=x \\
\text { end }(x, y)\end{array}$ & $\begin{array}{c}\text { program } \\
x:=0 \\
y:=x \\
z:=y \\
\text { end }(x, y, z)\end{array}$ & \\
\hline \multicolumn{2}{|c|}{$A-$ Base } & $A \cap$ Base $\cap B$ & $B-$ Base & $A[$ Base $] B$ \\
\hline \multicolumn{2}{|c|}{$\varnothing(=1)$} & $\begin{array}{c}\text { program } \\
x:=0 \\
\text { end }(x)\end{array}$ & $\begin{array}{c}\text { program } \\
x:=0 \\
y:=x \\
z:=y \\
\text { end }(z)\end{array}$ & $\begin{array}{c}\text { program } \\
x:=0 \\
y:=x \\
z:=y \\
\text { end }(x, z)\end{array}$ \\
\hline \multicolumn{2}{|c|}{$A \div$ Base } & $A \cup B a s e \cup B$ & $B \div$ Base & $A\{$ Base $\} B$ \\
\hline$G_{1}-\left\{s \in G_{1}\right.$ & $\begin{array}{c}\text { program } \leq s) \\
x:=0 \\
y:=x \\
\text { end }(0\end{array}$ & $\begin{array}{c}\text { program } \\
x:=0 \\
y:=x \\
z:=y \\
\text { end }(x, y, z)\end{array}$ & $G_{1}(=\mathrm{T})$ & $\begin{array}{c}\text { program } \\
x:=0 \\
\text { end }(x)\end{array}$ \\
\hline
\end{tabular}

Figure 2. An example for which $A\{$ Base $\} B \subset A[$ Base $] B$.

Because $A$ (Base $\} B$ is the intersection of $A \div$ Base with $(A \cup B a s e \cup B) \cap(B \div B$ ase $)$, none of the following three single-point slices, which are all members of $A[B$ ase $] B$, occur in $A\{$ Base $\} B$ :

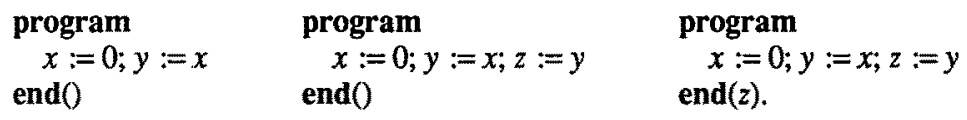

This example illustrates a fundamental difference between integrating by the operation $A[B a s e] B$ and integrating by $A\{$ Base $] B$. With $A[$ Base $] B$ an insertion made in one integrand can "override" a deletion in the other integrand; in the example shown above, the insertion of $z:=y$ in integrand $B$ overrides the deletion of $y:=x$ from integrand $A$. By contrast, with $A\{B$ ase $\} B$ a deletion in one integrand can override an insertion in the other integrand; in the example, the deletion of $y:=x$ from integrand $A$ overrides the insertion of $z:=y$ in integrand $B$. Consequently, we say that $A\{B$ ase $\} B$ is the deletionpreserving integration of $A$ and $B$ with respect to Base.

\section{COMPATIBLE INTEGRANDS}

Program integration deals with the problem of reconciling "competing" modifications to a base program. A different, but related, problem is that of separating consecutive edits to a base program into individual edits on the base program. Consider the case of two consecutive edits to base program $O$; let $O+\Delta A$ be the result of modifying $O$, and let $O+\Delta A+\Delta B$ be the result of modifying $O+\triangle A$. By "separating consecutive edits," we mean creating a program $O+\Delta B$ that includes the second modification but not the first. The algebraic framework introduced in the previous sections can be used in two different approaches to solving this problem. 


\subsection{Separating Consecutive Edits by Solving an Equation}

One way of formalizing our goal is to say that we are looking for an integrand $O+\Delta B$ that is compatible with base $O$, integrand $O+\Delta A$, and result $O+\Delta A+\Delta B$; that is, $O+\Delta B$ should satisfy the equation $(O+\Delta A)[O](O+\Delta B)=O+\Delta A+\Delta B$. Thus, our first approach poses the question "Given elements $a$, $b$, and $m$, when does there exist a solution for $x$ in the equation $a[b] x=m$ ?"

It is possible to show that if solutions to $a[b] x=m$ exist, they form a distributive lattice with a least element and a greatest element. Closed formulas can be given for the least and greatest elements.

THEOREM. Solutions of $a[b] x=m$ form $a$ distributive lattice with least element $(m \div a) \cup((a \cap b \cap m) \div(a \div b))$ and greatest element $m \cup(b \cap(m \div a))$.

Note that the formula for the greatest solution of $a[b] x=m$ makes use of the quotient operation, and thus holds only for double Brouwerian algebras.

\subsection{Separating Consecutive Edits by Re-Rooting}

It is also possible to take a different approach to the problem of separating consecutive edits. Consider again the case of two consecutive edits to a base program $O$, where $O+\triangle A$ is the result of modifying $O, O+\Delta A+\Delta B$ is the result of modifying $O+\Delta A$, and we want to create a program $O+\Delta B$ that includes the second modification but not the first. We can re-root the development history $O \rightarrow O+\Delta A \rightarrow O+\Delta A+\Delta B$ so that $O+\Delta A$, rather than $O$, is the base program. Programs $O$ and $O+\Delta A+\Delta B$ are treated as two variants of $O+\Delta A$. In this case, instead of treating the differences between $O$ and $O+\triangle A$ as changes that were made to $O$ to create $O+\Delta A$, they are now treated as changes made to $O+\Delta A$ to create $O$ : when $O$ is the base program, a statement $s$ that occurs in $O+\Delta A$ but not in $O$ is a "new" statement arising from an insertion; when $O+\Delta A$ is the base program, we treat the missing $s$ in $O$ as if a programmer had deleted $s$ from $O+\Delta A$ to create $O$. (The status of variant $O+\Delta A+\Delta B$ is unchanged; it is still treated as a variant derived from $O+\Delta A$.) $O+\Delta B$ is created by integrating $O$ and $O+\triangle A+\Delta B$ with respect to base program $O+\triangle A$, that is, by performing the integration $O[O+\Delta A](O+\Delta A+\Delta B)$.

The algebraic framework can be used to demonstrate that re-rooting and then integrating is reasonable in the sense that the program created in this way captures everything that is different between $O+\Delta A+\Delta B$ and $O+\Delta A$. Although in general the approach does not produce an integrand compatible with base $O$, integrand $O+\Delta A$, and result $O+\Delta A+\Delta B$ (even when a compatible integrand does exist), the element $E$ produced, where $E=O[O+\Delta A](O+\Delta A+\Delta B)$, has the property $(O+\Delta A)[O] E \supseteq O+\Delta A+\Delta B$. This property shows that $E$ captures everything that is different between $O+\Delta A+\Delta B$ and $O+\Delta A$ (as desired), plus more.

THEOREM. If $a[b] x=m$ has a solution for $x$, then $a[b](m[a] b) \supseteq m$.

\section{RELATION TO PREVIOUS WORK}

In unpublished work, Susan Horwitz and I found proofs of several algebraic properties of the HPR algorithm. The results given in this paper consist of the analogues for Brouwerian algebras of these earlier results, together with a number of new results. However, the method of proof used in this paper is much different than the proof techniques used to establish these earlier results, which involved complicated arguments-with many sub-cases and argument by reductio ad absurdum-about operations on dependence graphs. In contrast, the proofs given in this paper are strictly algebraic in nature, making use of the rich set of algebraic laws that hold in Brouwerian algebras.

The work described here was motivated by the desire to find a simpler way to prove properties about program integration. In this, I feel it succeeds-proofs in Brouwerian algebra are much less compli- 
cated than direct proofs about dependence graphs. It also provides a framework for studying common properties of variations on or extensions to the program-integration algorithm. The integration opera-

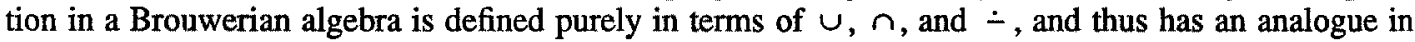
all Brouwerian algebras. Thus, to show that a proposed program-integration algorithm shares these properties, one merely has to show that the algorithm can be cast as an integration operation in a Brouwerian algebra.

The notation $a[b] c$ that has been used here for the integration operation in Brouwerian algebras is taken from a paper by Hoare in which he investigated some of the properties of $a[b] c$ in Boolean algebras [2]. However, nearly all of the questions examined in this paper (for Brouwerian algebras) were not addressed by Hoare (for Boolean algebras).

The integration operation $a[b] c$ in the Brouwerian dependence-graph algebra provides an alternative method for integrating programs to the HPR algorithm. The two methods do not have exactly the same properties. For example, in the dependence-graph algebra (as well as all other Brouwerian algebras), the operation $a[b] c$ satisfies the properties demonstrated in the paper; however, because the set of dependence graphs does not form a Brouwerian algebra, not all of the results established about integration in Brouwerian algebras hold for the HPR algorithm.

The remainder of this section compares the two program-integration methods. The bottom line is that, in addition to its elegant collection of algebraic properties, the operation $a[b] c$ in the dependence-graph algebra generalizes the HPR algorithm while preserving its most important property - the ability to characterize the execution behavior of the integrated program in terms of the behaviors of the base program and the two integrands.

\subsection{Interference Between Integrands}

In previous sections, we have not discussed the notion of interfering integrands. In the HPR algorithm, there are two possible ways in which the merged dependence graph $M$ can fail to represent a satisfactory integrated program; we refer to them as "Type I interference" and "Type II interference." The criterion for Type $I$ interference is based on a comparison of slices of the merged graph $M$ with the dependence graphs for programs $A$ and $B$. The slices $\Delta(A, B a s e)$ and $\Delta(B, B a s e)$ represent potentially changed computations of integrands $A$ and $B$ with respect to $B$ ase, respectively. Thus, there is interference if graph $M$ does not preserve these slices; that is, there is Type I interference if either $\Delta(A, B a s e)$ or $\Delta(B, B$ ase $)$ is not a slice of $M$.

The final step of the HPR algorithm involves reconstituting a program from dependence graph $M$. However, if there is no program whose dependence graph is $M$, we say there is Type II interference (and that graph $M$ is infeasible). (See [5] for a discussion of reconstructing a program from the merged dependence graph and the inherent difficulties of this problem.)

The class of integrations that are handled successfully by the integration operation in the Brouwerian dependence-graph algebra coincides with that handled by the HPR algorithm; however, the notion of interference is slightly different. Because the $U, n$, and - operations in the dependence-graph algebra can never "corrupt" slices, there is only one notion of interference in the dependence-graph algebra, namely, infeasibility; for an integration $a[b] c$, integrands $a$ and $c$ interfere if there is no program that corresponds to the element $a[b] c$. (This is not to say that it is any easier to test whether an element of the dependence-graph algebra is feasible than it is to test whether an individual dependence graph is feasible, as is done in the HPR algorithm; we are simply pointing out that for integration in the dependence-graph algebra, there is only one notion of interference instead of two.)

Another difference between the two integration algorithms that relates to interference is that in the dependence-graph algebra infeasible elements are subject to the same algebraic laws as feasible elements, which can have certain advantages. For example, when performing a succession of integrations to propagate a change through the development history of a program, some of the intermediate elements produced may be infeasible (indicating interference). Yet subsequent integrations that involve 
these infeasible elements may, in fact, result in feasible elements. Because Type I interference indicates that a slice has been corrupted, it is not meaningful to perform the analogous integrations with the HPR algorithm.

\subsection{Semantic Properties of the Integrated Program}

One of the virtues of the HPR algorithm is that there is a theorem that characterizes the execution behavior of the integrated program produced by the HPR algorithm in terms of the behaviors of the base program and the two integrands.

THEOREM. (INTEGRATION THEOREM [11]). If programs $A$ and $B$ are two non-interfering variants of Base, and program $M$ is the result of integrating $A$ and $B$ with respect to Base (via the HPR algorithm), then for any initial state $\sigma$ on which $A, B$, and Base all halt,

(1) $M$ halts on $\sigma$.

(2) If $x$ is a variable defined in the final state of $A$ for which the final states of $A$ and Base disagree, then the final state of $M$ agrees with the final state of $A$ on $x$.

(3) If $y$ is a variable defined in the final state of $B$ for which the final states of $B$ and Base disagree, then the final state of $M$ agrees with the final state of $B$ on $y$.

(4) If $z$ is a variable on which the final states of $A, B$, and Base agree, then the final state of $M$ agrees with the final state of Base on $z$.

For the HPR algorithm, the Integration Theorem follows from two theorems about slices. One, the Slicing Theorem, which was stated in Section 2, demonstrates that a slice captures a portion of a program's behavior in the sense that, for any initial state on which the program halts, the program and the slice compute the same sequence of values for each element of the slice. The other, the Termination Theorem, demonstrates that if a program is decomposed into (two or more) slices, the program halts on any state for which all the slices halt.

THEOREM. (TERMINATION THEOREM [11]). Let $P$ be a program. Suppose $X$ and $Y$ are sets of vertices such that $G_{P}=G_{P} / X \cup G_{P} / Y$. If $P / X$ and $P / Y$ halt on a state $\sigma$, then $P$ halts on $\sigma$ as well.

Note that the Termination Theorem and clause (1) of the Slicing Theorem are complementary: clause (1) of the Slicing Theorem asserts that if a program terminates then each slice also terminates; the Termination Theorem asserts that when a program can be decomposed into two slices, if each slice terminates then the program terminates. We can then apply clause (2) of the Slicing Theorem to conclude that the two slices (collectively) compute the same sequence of values as the entire program.

It follows from the Slicing Theorem, the Termination Theorem, the definition of the dependencegraph algebra, and the definition of the operation $A[B$ ase $] B$ that the Integration Theorem also holds for $A[$ Base $] B$ in the dependence-graph algebra.

\subsection{Integration Without Tags}

In the HPR algorithm, it is assumed that a specialized program editor-providing a tagging capability so that common components can be identified in different versions-is used to create the program variants from the base program. This assumption can be relaxed; that is, two different examples of Brouwerian algebras that can be used for integrating programs are (1) the set of downwards-closed sets of tagged single-point slices, and (2) the set of downwards-closed sets of untagged single-point slices.

It must be noted that the two different definitions of dependence-graph algebras correspond to two different variations on the HPR algorithm. However, although the integration algorithm based on sets of untagged slices produces a somewhat different answer than the algorithm based on sets of tagged slices (both in terms of the final program that is the result of an integration, as well as in the notion of when an integration fails due to interference), the Integration Theorem still holds. That is, for both dependence-graph algebras and the HPR algorithm, we have the same characterization of the execution 
behavior of the integrated program in terms of the execution behaviors of the base program and the two integrands.

The benefits of doing without tags are two-fold. First, it is no longer necessary for program integration to be supported by a closed system; in principle, programs can be integrated even if they are created using ordinary text editors. Second, the class of integration problems that can be handled successfully in the dependence-graph algebra based on sets of untagged slices is strictly larger than the class that can be handled by the algebra based on tagged slices (which coincides with the class handled by the HPR algorithm).

\section{IMPLEMENTATION}

A program-integration tool that uses the HPR algorithm has been demonstrable since the summer of 1987 [12]. The integration tool is embedded in a program editor created using the Synthesizer Generator, a meta-system for creating interactive, language-based program development systems [10]. The editor automatically supplies tags on program elements so that common statements and predicates can be identified in different versions. Data-flow analysis of programs is carried out according to the editor's defining attribute grammar and used to construct dependence graphs. An integration command added to the editor invokes the integration algorithm on the dependence graphs, reports whether the variant programs interfere, and, if there is no interference, builds the integrated program.

This implementation has recently been extended to incorporate the ideas described in this paper; we added an editor and interpreter for a higher-order functional language that operates on sets of tagged slices (SLICE_SETs). The primitive operations on SLICE_SETs are join, meet, pseudo-difference, and integration. Functional expressions are built up using lambda-abstraction, application, conditional expressions, and let-clauses. A free variable in an expression (say $x$ ) denotes the SLICE_SET created from the program in editing buffer $x$. If no such buffer exists, the value is $\perp$. An evaluation command added to the editor invokes the interpreter on the expression, and-if the final result is a SLICE_SET-builds the corresponding program (if one exists).

\section{APPENDIX: ALGEBRAIC LAWS FOR BROUWERIAN ALGEBRAS}

This appendix covers algebraic laws that hold for Brouwerian algebras. The material presented here is meant to make the paper self-contained; further information about Brouwerian algebras can be found in [7]. (The alge-

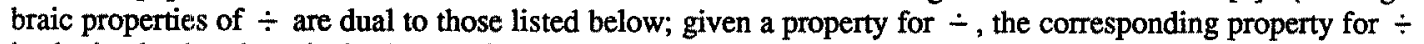
is obtained using the substitution: $\cup$ for $\cap, \cap$ for $\cup, \div$ for,$- \subseteq$ for $\supseteq, \supseteq$ for $\subseteq$, and "max" for "min.")

\section{Fundamental Properties of -}

(a) $a-b \subseteq c$ iff $a \subseteq b \cup c$

\section{Algebraic Properties of -}

The following properties of Brouwerian algebras are taken from [9] (pp. 59-60); the numbering for laws (4) - (24) follows that given in [9]. (In [9], the laws are expressed in dual form, using a "pseudo-complement" operator, rather than in the form given below, where pseudo-difference is used.)
(4) $b-a=\perp$ iff $a \supseteq b$
(6) $a \div a=1$
(5) $a=b$ iff $b-a=\perp=a-b$
(8) $b-1=b$
(7) $1-a=1$
(10) $a \cup(b-a) \supseteq b$
(12) If $b_{1} \supseteq b_{2}$ then $b_{1}-a \supseteq b_{2}-a$
(9) $(a-a) \cup b=b$
(14) $a \cup(b-a)=a \cup b$
(11) If $a_{1} \supseteq a_{2}$ then $b-a_{2} \supseteq b-a_{1}$
(13) $b \geq 2 \div a$
(16) $(b-a) \cup(c-a)=(b \cup c)-a$
(18) $(c \dot{-b)}-a=c \div(a \cup b)=(c \dot{-} a) \dot{-} b$
(15) $(b-a) \cup b=b$
(17) $(c-a) \cup(c \dot{-} b)=c \div(a \cap b)$
(19) $a-c 2(b-c)-((b-a) \div c)$. 
(20) $(b-a) \cup(c-b) \supseteq c-a$
(22) $a \supseteq(a \cup b) \div b$
(24) $c \cup((c \cup b) \div(c \cup a))=c \cup(b-a)$

(21) $(b-a) 2(c-a) \div(c-b)$

(23) $(c-b)-a \geq(c-a)-(b-a)$

Additional Algebraic Properties of -

The following properties of Brouwerian algebras are taken from [13].

(25) $(a-b) \div(a \cap b)=a \div b$

(26) $(b-a) \cup(b \cap a)=b$

(27) $(c-b)-a=(c-a) \div(b-a)$

(28) $(a \cap b)-c \subseteq a \cap(b-c)$

(29) $(a \cap b)-c \subseteq(a-c) \cap(b-c)$

(30) $b \div(b-a) \subseteq a \cap b$

(31) If $a-b \subseteq b$, then $a \subseteq b$

(32) $c-(a \cup b) \subseteq(c-a) \cap(c-b)$

(33) $(a-b) \div(b-a)=a-b$

(34) $(a \cup b) \div(a \cap b)=(a-b) \cup(b-a)$

\section{ACKNOWLEDGEMENTS}

Tony Hoare suggested using the notation $a[b] c$ for the integration operation and pointed out the existence of [2], which discusses properties of $a[b] c$ in Boolean algebras. Susan Horwitz provided many comments and helpful suggestions.

\section{REFERENCES}

1. Ferrante, J., Ottenstein, K., and Warren, J., "The program dependence graph and its use in optimization," ACM Transactions on Programming Languages and Systems 9(3) pp. 319-349 (July 1987).

2. Hoare, C.A.R., "A couple of novelties in the propositional calculus," Zeitschr. f. math. Logik und Grundlagen $d$. Math. 31 pp. 173-178 (1985).

3. Horwitz, S., Reps, T., and Binkley, D., "Interprocedural slicing using dependence graphs," Proceedings of the ACM SIGPLAN 88 Conference on Programming Language Design and Implementation, (Atlanta, GA, June 22-24, 1988), ACM SIGPLAN Notices 23(7) pp. 35-46 (July 1988).

4. Horwitz, S., Pfeiffer, P., and Reps, T., "Dependence analysis for pointer variables," Proceedings of the ACM SIGPLAN 89 Conference on Programming Language Design and Implementation, (Portland, OR, June 21-23, 1989), ACM SIGPLAN Notices 24(7) pp. 28-40 (July 1989).

5. Horwitz, S., Prins, J., and Reps, T., "Integrating non-interfering versions of programs," ACM Trans. Program. Lang. Syst. 11(3) pp. 345-387 (July 1989).

6. Kuck, D.J., Kuhn, R.H., Leasure, B., Padua, D.A., and Wolfe, M., "Dependence graphs and compiler optimizations;" pp. 207-218 in Conference Record of the Eighth ACM Symposium on Principles of Programming Languages, (Williamsburg, VA, January 26-28, 1981), ACM, New York, NY (1981).

7. McKinsey, J.C.C. and Tarski, A., "On closed elements in closure algebras," Annals of Mathematics 47(1) pp. 122-162 (January 1946).

8. Ottenstein, K.J. and Ottenstein, L.M., "The program dependence graph in a software development environment," Proceedings of the ACM SIGSOFTISIGPLAN Software Engineering Symposium on Practical Software Development Environments, (Pittsburgh, PA, Apr. 23-25, 1984), ACM SIGPLAN Notices 19(5) pp. 177-184 (May 1984).

9. Rasiowa, H. and Sikorski, R., The Mathematics of Metamathematics, Polish Scientific Publishers, Warsaw (1963).

10. Reps, T. and Teitelbaum, T., The Synthesizer Generator: A System for Constructing Language-Based Editors, Springer-Verlag, New York, NY (1988).

11. Reps, T. and Yang, W., "The semantics of program slicing," TR-777, Computer Sciences Department, University of Wisconsin, Madison, WI (June 1988).

12. Reps, T., "Demonstration of a prototype tool for program integration," TR-819, Computer Sciences Department, University of Wisconsin, Madison, WI (January 1989).

13. Reps, T., "On the algebraic properties of program integration," TR-856, Computer Sciences Department, University of Wisconsin, Madison, WI (June 1989).

14. Weiser, M., "Program slicing," IEEE Transactions on Software Engineering SE-10(4) pp. 352-357 (July 1984).

15. Yang, W., Horwitz, S., and Reps, T., "Detecting program components with equivalent behaviors," TR-840, Computer Sciences Department, University of Wisconsin, Madison, WI (April 1989).

16. Yang, W., Horwitz, S., and Reps, T., "A new program integration algorithm," TR-899, Computer Sciences Department, University of Wisconsin, Madison, WI (December 1989). 